\title{
The Existence of Maximal Slicings in Asymptotically Flat Spacetimes
}

\author{
M. Cantor ${ }^{\star}$, A. Fischer ${ }^{\star \star \star \star \star}, J$. Marsden ${ }^{+\star \star \star}$, N. Ō Murchadha ${ }^{++}$, \\ and J. York ${ }^{++}$ \\ Department of Physics and Astronomy, University of North Carolina, \\ Chapel Hill, North Carolina 27514, USA
}

\begin{abstract}
We consider Cauchy data $(g, \pi)$ on $\mathbb{R}^{3}$ that are asymptotically Euclidean and that satisfy the vacuum constraint equations of general relativity. Only those $(g, \pi)$ are treated that can be joined by a curve of "sufficiently bounded" initial data to the trivial data $(\delta, 0)$. It is shown that in the Cauchy developments of such data, the maximal slicing condition $\operatorname{tr} \pi=0$ can always be satisfied. The proof uses the recently introduced "weighted Sobolev spaces" of Nirenberg, Walker, and Cantor.
\end{abstract}

Consider the set $\mathscr{C}$ of spacetimes which are the Cauchy developments of initial data $(g, \pi)$ on $\mathbb{R}^{3}$ which are asymptotically Euclidean and which satisfy the constraint equations [see (3) and (4) below] in the dynamical formulation of general relativity [1]. In 1968, Brill and Deser [2] conjectured that one can maximally slice any such spacetime, i.e. one can find spacelike hypersurfaces on which $\operatorname{tr} \pi=0$. In a Hamiltonian analysis of general relativity $\operatorname{tr} \pi$ assumes the role of a gauge variable (see for example [12]) and so one would expect that the $\operatorname{tr} \pi=0$ condition can be met in any such spacetime. Here we prove that the Brill-Deser conjecture is true.

We consider only those $(g, \pi)$ which can be joined by a curve of "sufficiently bounded" initial data (to be explained later) to flat space $(\delta, 0)$. Thus we are considering the component $\mathscr{C}_{0}$ of $(\delta, 0)$ in the set of asymptotically Euclidean solutions of the constraint equations. $\mathscr{C}_{0}$ is restricted to those 3 -metrics which are derived from Lorentz metrics on $\mathbb{R}^{4}$ that are near the "background" Minowski metric. The set $\mathscr{C}_{0}$ is discussed in [7-11].

* Present address: Department of Mathematics, Duke University, Durham, North Carolina 27706, USA

$\star \star$ Present address: Department of Mathematics, University of California, Santa Cruz, California 95064, USA

$\star \star \star$ Research partially supported by National Science Foundation Grants GP-39060 and GP-15735

+ Present address: Department of Mathematics, University of California, Berkeley, California 94720, USA

++ Research partially supported by National Science Foundation Grant GP-43909 to the University of North Carolina 
In her note [6], Choquet-Bruhat proves a theorem for spacetimes with compact spacelike hypersurfaces which is similar to our step 2 below. She also notes her proof extends to yield the local result for spacetimes with noncompact spacelike hypersurfaces. The authors became aware of [6] after the present work was completed.

We shall prove:

Theorem. Let $(g, \pi) \in \mathscr{C}_{0}$. Then in the Cauchy development of $(g, \pi)$ there is a slice on which the trace of the second fundamental form is zero. (Recall that this entails $\operatorname{tr} \pi=0)$.

There is a similar theorem for the component of $\mathscr{C}$ containing any given $(g, \pi)$ with $\operatorname{tr} \pi=0$ or in the case of compact hypersurfaces, $\operatorname{tr} \pi / \mu_{g}=$ constant (see [6] and [12]). The constant depends on the hypersurface. This theorem is proven similarly to the one in this paper.

The proof requires the use of the weighted Sobolev spaces $M_{s . \delta}^{p}$ introduced in [3]. For compact hypersurfaces, the usual Sobolev spaces $W^{s, p}$ will do, as in $[9]$.

Definition. Let $\sigma(x)=\left(1+|x|^{2}\right)^{1 / 2}$. For $1 \leqq p \leqq \infty, s$ a nonnegative integer, and $\delta \in \mathbb{R}$, let $M_{s . \delta}^{p}\left(\mathbb{R}^{n}, \mathbb{R}^{q}\right)$ be the completion of $C_{0}^{\infty}\left(\mathbb{R}^{n}, \mathbb{R}^{q}\right)$ with respect to the norm

$$
|f|_{p . s . \delta}=\sum_{|\alpha| \leqq s}\left(\left|\left(D^{\alpha} f\right) \sigma^{\delta+|\alpha|}\right|_{L^{p}}\right) .
$$

The elementary properties of these spaces are discussed in $[3,4]$.

The important technical result for this paper is

Lemma 1. [5]. Let $n>m$ and $A_{\infty}=\sum_{|\alpha|=m} \bar{a}_{\alpha} D^{\alpha}$ be an elliptic homogeneous operator on $\mathbb{R}^{n}$. Suppose we have an elliptic operator $A=\sum_{|\alpha| \leqq m} a_{\alpha}(x) D^{\alpha}$ on $\mathbb{R}^{n}$ satisfying for $s \geqq m, a_{\alpha} \in C^{s-m}$ and

$$
\sup \left|D^{\gamma}\left(a_{\alpha}(x)\right) \cdot \sigma^{m-\alpha+|\gamma|}\right|<\infty \quad \text { for } \quad|\alpha|<m
$$

and

$$
\lim \sup \left|D^{\gamma}\left(a_{\alpha}(x)-\bar{a}_{\alpha}\right) \sigma^{|\gamma|}\right|<\varepsilon \quad \text { for } \quad|\alpha|=m
$$

and $|\gamma| \leqq s-m$. Then if $p>n /(n-m)$ and $0 \leqq \delta<-m+n(p-1) / p$, and $\varepsilon$ is sufficiently small, $\bar{A}$ is an isomorphism between $M_{s . \delta}^{p}$ and $M_{s-m . \delta+m}^{p}$.

Remark. The smoothness condition of the $a_{\alpha}$ may be relaxed by taking completions in the appropriate Banach space of linear operators. This fact is used implicitly below.

We shall apply Lemma 1 where $A$ is the Laplacian with respect to some asymptotically flat metric on $\mathbb{R}^{3}$. Thus $n=3$ and $m=2$. We assume $p$ and $\delta$ are as in the theorem and $s>n / p+2$. The $(g, \pi)$ we shall consider will be of the form $g=\delta+h$ with $h \in M_{s . \delta}^{p}$ and $\pi \in M_{s-1 . \delta+1}^{p}$ (see [8]). All norms are taken with respect to the flat background metric. Note that for $g \in \mathscr{C}_{0}$ these norms are equivalent to those induced by $g$. Note we may take $\operatorname{tr} \pi \in M_{s-1 . \delta+2}^{p}$. The topology on the space of initial data is given by the $M_{s, \delta}^{p}$ norms. 
The required slicing will be determined by a lapse function $N(\lambda, x)=\left(-g^{00}\right)^{-1 / 2}$. Letting the shift vector $g_{0 i}=X_{i}(\lambda, x)=0$, the Einstein. Equations read (here $\pi$ is a density):

$$
\begin{aligned}
& \partial g / \partial \lambda= 2 N\left(\pi-\frac{1}{2}(\operatorname{tr} \pi) g\right)\left(1 / \mu_{g}\right)=-2 N k \\
& \begin{aligned}
\partial \pi / \partial \lambda= & -N\left(\operatorname{Ric}(g)-\frac{1}{2} R(g) g\right) \mu_{g}+\frac{1}{2} N\left(\pi \cdot \pi-\frac{1}{2}(\operatorname{tr} \pi)^{2}\right) / \mu_{g} \\
& -2 N\left(\pi \times \pi-\frac{1}{2}(\operatorname{tr} \pi) \pi\right) / \mu_{g}+\left(\operatorname{Hess} N-g \nabla^{2} N\right) \mu_{g}
\end{aligned} \\
& \begin{aligned}
\mathscr{H}(g, \pi)=\left(\pi \cdot \pi-\frac{1}{2}(\operatorname{tr} \pi)^{2}\right) / \mu_{g}-R(g) \mu_{g}=0 \\
\delta_{g} \pi=0
\end{aligned}
\end{aligned}
$$

and using $p=\operatorname{tr} \pi / \mu_{g}=2 \operatorname{tr} k$, we find from the above equations that

$$
\partial p / \partial \lambda=2\left(k \cdot k-\nabla^{2}\right) N \text {. }
$$

Step. 1. If $p=0$ for some $\lambda$, we may choose an $N$ such that $p$ is zero for all $\lambda$ (for which the dynamics is defined).

Proof. Writing $N=1+\tilde{N}$ (so that $\tilde{N}$ is close to 0 when $N$ is close to 1 ), we find

$$
\partial p / \partial \lambda=2 k \cdot k+2\left(k \cdot k-\nabla^{2}\right) \tilde{N} .
$$

Thus the equation $\partial p / \partial \lambda=0$ may be solved using Lemma 1 for $\tilde{N}(\lambda) \in M_{s+1}^{p} \delta$ for each $\lambda$. Thus for this choice of $N=1+\tilde{N}$ in the dynamics the condition $p=0$ will be maintained.

In what follows we show that whatever $p$ equals at $\lambda=0$, we may achieve $p=0$ at $\lambda=1$ by choosing a suitable $N$. Throughout, we shall take $\partial N / \partial \lambda=0$.

Step 2. (Local Argument). Let $\left(g_{0}, \pi_{0}\right) \in \mathscr{C}_{0}$ and suppose $\operatorname{tr} \pi_{0}=0$. Then there is a neighborhood $V$ of $\left(g_{0}, \pi_{0}\right)$ such that if $(g, \pi) \in V$ then there is an $N \in \mathscr{M}_{s+1, \delta}^{p}$ such that $p=0$ at $\lambda=1$. (By a suitable choice of scale, we may assume $\lambda=1$ will be reached by the dynamics.)

Proof. Let $F=\mathscr{C}_{0} \times \mathscr{M}_{s+1, \delta}^{p}\left(\mathbb{R}^{3}, \mathbb{R}\right) \rightarrow M_{s-1, \delta+2}^{p}\left(\mathbb{R}^{3}, \mathbb{R}\right)$ be defined (on a suitable open set) by

$$
F((g, \pi), N)=\{\text { the function } p \text { at } \lambda=1 \text { determined by Equations (1), (2), (5) }\} \text {. }
$$

Then using smoothness properties of the evolution equations (see [8]), $F$ is a smooth mapping. The derivative with respect to $N$ at $\left(\left(g_{0}, \pi_{0}\right), 0\right)$ in the direction $\delta N$ is

$$
D_{N} F\left(\left(g_{0}, \pi_{0}\right), 0\right) \cdot \delta N=\left(\int_{0}^{1}\left(k_{0}(\lambda) \cdot k_{0}(\lambda)-\nabla_{\lambda}^{2}\right) d \lambda\right) \delta N
$$

where $k_{0}(\lambda)$ is the evolution of $k_{0}$ for the given $\left(g_{0}, \pi_{0}\right)$ and $\nabla_{\lambda}^{2}$ is the Laplacian for $g_{0}(\lambda)$.

Since we are only considering functions that are independent of $\lambda$, it follows easily from Lemma 1 that the operator (6) is an isomorphism (see also [9]). Thus by the implicit function theorem we can uniquely solve $F((g, \pi), N)=0$ for $N(g, \pi)$ near 0 and $(g, \pi)$ near $\left(g_{0}, \pi_{0}\right)$. This proves step 2 . 
Step 3 (Globalization). Let $\left(g_{0}, \pi_{0}\right)$ be joined to $(g, \pi)$ be a continuous curve $(g(\alpha), \pi(\alpha))$ in $\mathscr{C}_{0}, \alpha \in[0,1]$. Let $J$ be the set of $\alpha$ for which the resulting space time has a maximal slice. Then $0 \in J$ and step 2 shows that $J$ is open. We can always work in a neighborhood of the curve $(g(\alpha), \pi(\alpha))$ so that the evolution times used in step 2 , can be chosen to be uniform along the curve.

To show $J$ is closed, let $\alpha_{m} \in J$ and $\alpha_{m} \rightarrow \alpha$. Let $N_{m}$ be the unique lapse functions given by step 2 . In order to demonstrate that $\nabla_{m}^{2}$ remains uniformly elliptic and the slices "uniformly spacelike", we may take a sequence of coordinate transformations $f_{m}$ on the slices $S_{m}$ chosen so as to keep the eigenvalues of $g_{m}$ (relative to the flat background metric) bounded away from zero. Since $k_{0}(m)$ remains uniformly bounded and $\nabla_{m}^{2}$ remains uniformly elliptic for $\alpha_{m}, m \rightarrow \infty$, the $N_{m}$ will converge to a function $N$. This $N$ is the required zero of $F$.

Thus $J=[0,1]$ and our proof is complete.

Note Added in Proof. The hypotheses of Lemma 1 should include that $a_{0}$ is non-positive. In our application, $a_{0}=-\int_{0}^{1} k_{0}(\lambda) \cdot k_{0}(\lambda) d \lambda \leqq 0$.

\section{References}

1. Arnowitt, R., Deser,S., Misner,C.W.: The dynamics of general relativity, In: Gravitation: an introduction to current research, (ed. L. Witten). New York: Wiley 1962

2. Brill,D., Deser,S.: Variational methods and positive energy in general relativity. Ann. Phys. 50, $548-570(1968)$

3. Cantor, M.: Spaces of functions with asymptotic conditions on $\mathbb{R}^{n}$. Ind. U. J. Math. to appear (1975)

4. Cantor, M.: Perfect fluid flows over $\mathbb{R}^{n}$ with asymptotic conditions, J. Funct. Anal. 18, $73-84$ (1975)

5. Cantor, M.: Growth of Solutions of elliptic equations with nonconstant coefficients on $\mathbb{R}^{\prime \prime}$. Preprint

6. Choquet-Bruhat, Y.: Sous-Varietes maximales, ou a courbure constante, de varietes lorentziennes, C. R. Acad. Sc. Paris, 280, Ser A, 169-171 (1975)

7. Fischer, A., Marsden, J.: The Einstein equations of evolution - A geometric approach. J. Math. Phys. 13, 546-568 (1972)

8. Fischer,A., Marsden, J.: The Einstein evolution equations as a first order quasi-linear hyperbolic system. Commun. math. Phys. 28, 1-38 (1972)

9. Fischer, A., Marsden, J.: Linearization stability of nonlinear partial differential equations, Proc. Symp. Pure Math. A. M. S. 27, 219-263 (1975) (also Bull. A. M. S. 79, 997-1003 (1973), 80, 479-484, and General Relativity and Gravitation 5, 73-77 (1974)

10. O'Murchadha, N., York, J. W.: Initial value problem of general relativity (I, II). Phys. Rev. D 10, $428-436,437-446(1974)$

11. O’Murchadha, N., York, J.W.: Gravitational energy. Phys. Rev. D 10, 2345-2357 (1974)

12. York, J.W.: The role of conformal three geometry in the dynamics of gravitation. Phys. Rev. Letters 28, 1082 (1972)

Communicated by J. Ehlers

Received July 9, 1975 